Casos Clínicos

Arch. Esp. Urol., 61, 4 (544-546), 2008

\section{MELANOMA EN GLANDE, A PROPÓSITO DE UN CASO}

José Manuel Janeiro Pais, Vicente Pastor Casas Agudo, Daniel López García, José Ponce Díaz-Reixa, Cipriano Lamas Meilán y Marcelino González Martín.

Servicio de Urología. Hospital Juan Canalejo. La Coruña. España.

Resumen.- OBJETIVO: Presentar un nuevo caso de melanoma localizado en el glande.

MÉTODOS/RESULTADOS: Presentamos en este artículo el caso de un paciente diagnosticado y tratado en nuestro servicio, describiendo su forma de presentación así como su manejo terapéutico.

CONCLUSIONES: El melanoma de localización peneana es una forma infrecuente de presentación de este tipo de tumores cutáneos. Hacemos especial hincapié en su diagnóstico, presentación clínica y manejo terapéutico, ya que se trata de una neoplasia que requiere un diagnóstico y tratamiento precoces debido a su pronta diseminación metastásica
José Manuel Janeiro Pais

Calle Lázaro Cárdenas, 23 - 1 E.

15009 La Coruña. (España)

janeiropais@canalejo.org

Trabajo recibido: 11 de agosto 2007
Palabras clave: Melanoma. Glande.

Summary.- OBJECTIVE: To report one case of me-lanoma of the glans penis.

METHODS/RESULTS: We present the case of a patient diagnosed and treated in our department describing diagnosis and therapeutic management.

CONCLUSIONS: Melanoma of the penis is an uncommon presentation form of this kind of cutaneous tumor. We especially emphasize its diagnosis, clinical presentation and therapeutic management due to its prompt metastatic dissemination requiring early diagnosis and treatment.

Keywords: Melanoma. Glans penis.

\section{INTRODUCCIÓN}

El melanoma de pene es una rara entidad tumoral de la que apenas hay recogidos 200 casos en la literatura, representando alrededor del $1 \%$ de los tumores malignos que asientan sobre los genitales externos y menos del $1 \%$ de todos los melanomas, siendo su incidencia inferior al $1 \%$ en la población general.

La localización mas frecuente de estas lesiones es el glande, pudiendo asentar con menor frecuencia a nivel de prepucio, meato uretral, surco balanoprepucial y resto del pene, siendo esto último lo menos frecuente.

La edad de presentación, a diferencia de melanomas de otras localizaciones anatómicas, que aparecen a edades mas tempranas, suele ser entre la sexta y octava décadas de la vida.

Su forma de presentación es variable, aunque habitualmente asientan sobre lesiones preexistentes que han crecido o se han ulcerado recientemente y que sangran con facilidad al roce $(1,2)$. En otras ocasiones se manifiestan como una lesión de aparición reciente, plana y de contornos irregulares o bien de aspecto nodular y de coloración irregular marrón o negruzca (3).

\section{CASO CLÍNICO}

Presentamos el caso de un varón de 70 años con antecedentes de gastrectomía por adenocarcinoma gástrico hace 12 años y carcinoma de próstata Gleason VII, (2 cilindros de 6 afectados por el tumor), tratado quirúrgicamente mediante una prostatectomía radical hace diez años, con un ganglio linfático afectado por el tumor y bloqueo androgénico hasta la actualidad, con PSA de 0,06 en la ultima revisión. Acude al Servicio de Urgencias de nuestro hospital por presentar una lesión exofíti- 
ca no dolorosa a nivel del glande de unas cinco semanas de evolución. No recordaba haber tenido ninguna lesión previa ni traumatismo en la zona, y refería desde la aparición de la lesión algún episodio de sangrado con el roce (Figura 1).

En la exploración del paciente observamos en el dorso del glande una lesión redondeada exofítica de color rojizo oscuro de aproximadamente dos centímetros de diámetro, con tendencia al sangrado, consistencia elástica y no dolorosa. No se palpaban linfadenectomías y el resto de la exploración física del paciente fue normal.

Se realizó biopsia de la lesión, observándose durante la intervención que se trataba de una tumoración de aspecto macroscópico no infiltrante, asentanda en la mucosa del glande, con áreas de aspecto trombosado y fácilmente friables (Figura2).

El análisis anatomopatológico intraoperatorio fue informado como lesión compuesta por células indiferenciadas con hipercromatismo nuclear, pleomorfismo y gran número de mitosis y pigmento negruzco, que sería compatible con el diagnóstico de melanoma.

Se completó la resección con una penectomía subtotal con cuatro centímetros de margen de seguridad desde el borde de la lesión y una linfadenectomía inguinal superficial bilateral con ganglios no afectados por el tumor. Se realizó TAC tóracoabdominal que mostró ausencia de enfermedad metastásica. El estudio anatomopatológico de la pieza penectomía reflejo una profundidad máxima de invasión de $1,5 \mathrm{~mm}$. El análisis inmunohistoquímico con S-100 y HMB 45 confirmó el diagnóstico de melanoma (1).

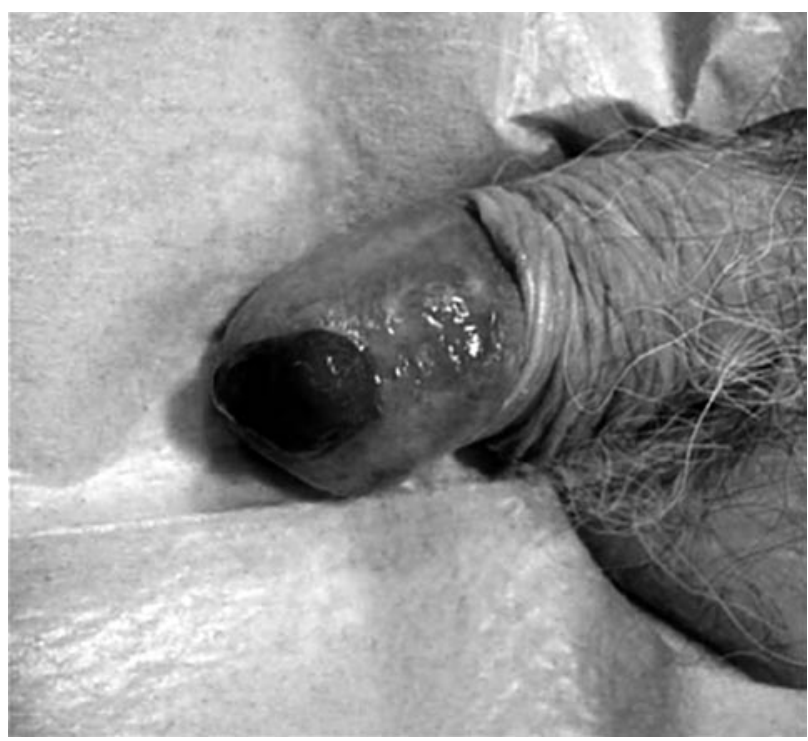

FIGURA 1. Lesión a su llegada a urgencias.

\section{DISCUSIÓN}

El retraso diagnostico que se produce en gran parte de los pacientes debido bien a la reticencia de los mismos a la hora de consultar este tipo de lesiones o bien a la ausencia de sospecha clínica por parte del facultativo unido a su temprana diseminación local, a ganglios linfáticos superficiales y profundos y a distancia por vía hematógena hace que el pronóstico de estos tumores sea sombrío en la mayor parte de los casos (3).

Existen distintos sistemas de estadiaje que nos permiten establecer niveles pronósticos en el melanoma peneano, entre los cuales cabe destacar por sus implicaciones terapéuticas la clasificación de Bracken y Diokno que divide la enfermedad en tres estadios desde el punto de vista anatomoclínico (4):

- Estadio I: Enfermedad limitada al pene.

- Estadio II: Afectación de ganglios linfáticos inguinales.

- Estadio III: Metástasis a distancia.

Y la clasificación de Breslow, que desde un punto de vista histológico diferencia cinco niveles de afectación cutánea según la profundidad de la lesión en milímetros:

- $<$ de $0,75 \mathrm{~mm}$

$-0,76-1,49 \mathrm{~mm}$

$-1,50-2,49 \mathrm{~mm}$

$-2,50-3,99 \mathrm{~mm}$

- > $4 \mathrm{~mm}$

El nivel de invasión anatómica se refleja en la clasificación de Clarck, siendo el estadío I cuando afecta sólo a la epidermis, el II a la dermis papilar, el III a la dermis reticular parcialmente, el IV a la dermis reticular por

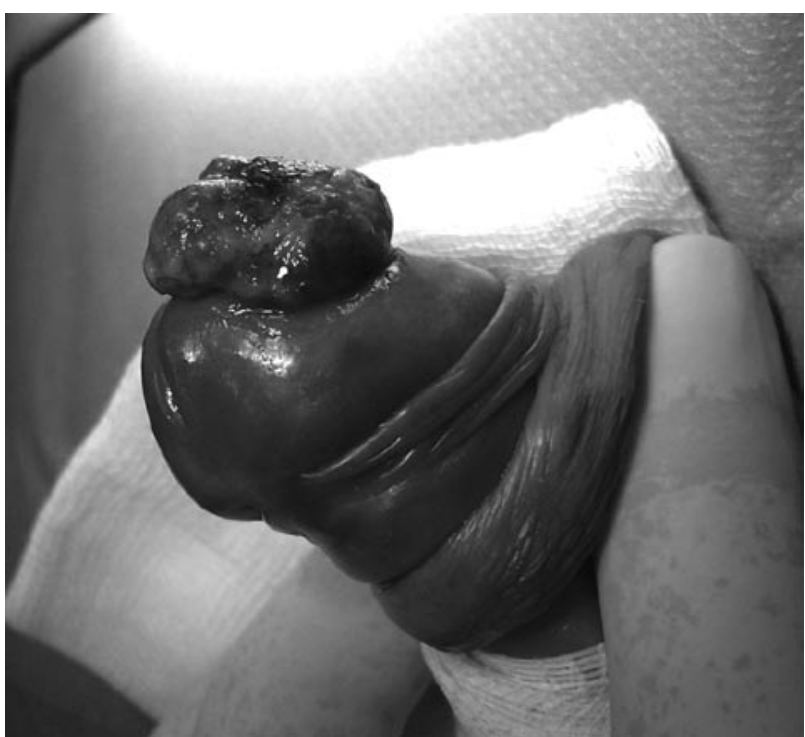

FIGURA 2. Aspecto de la lesión antes de la cirugía. 
completo y el nivel $V$ cuando afecta a la hipodermis y tejido graso subcutáneo.

El único tratamiento con intención curativa de estos tumores es el quirúrgico, el cual unicamente es eficaz cuando la enfermedad se encuentra limitada al pene (Estadio I de la clasificación de Bracken y Diokno). La cirugía debe consistir en la penectomía parcial con un margen de seguridad suficiente que será de entre tres - cinco centímetros para tumores con una profundidad de más de un milímetro en la clasificación de Breslow y 1 a 3 $\mathrm{cm}$ en tumores que no alcancen esa profundidad. Cuando la lesión tiene una profundidad superior a $1,5 \mathrm{~mm}$ es aconsejable realizar en el mismo acto linafadenectomía de los ganglios inguinales superficiales debido al mayor riesgo de diseminación metastásica en estos (2).

La linfadenectomía inguinal bilateral debe realizarse igualmente en pacientes con ganglios palpables (Estadio dos de Bracken y Diokno) para su correcto estadiaje (5).

\section{CONCLUSIONES}

El melanoma de localización peneana es una patología infrecuente con un pronostico ominoso en la mayor parte de los pacientes debido casi siempre al retraso diagnostico de la lesión (6).

La rápida diseminación linfática y hematógena (Estadios II - III) hace que estos tumores tengan un porcentaje de supervivencia a los 5 años cercano a cero con los trata- mientos disponibles en la actualidad como la inmunoterapia basada en el Interferón y la quimioterapia (7).

Por todo ello ante cualquier lesión sospechosa en el aparato genital es imprescindible un diagnostico precoz mediante biopsia, actuando sin demora en caso de confirmarse una lesión de este tipo.

\section{BIBLIOGRAFÍA y LECTURAS RECOMENDADAS (*lectura de interés $y^{* *}$ lectura fundamental)}

**1. BLANCO DIEZ, A.; MONTES COUCEIRO, M.; ALVAREZ CASTELO, L.y cols.: "Penile Melanoma: Clinical Case". Actas Urol. Esp., 26: 143, 2002.

*2. PARRA MUNTANER, L.; MADRID GARCIA, F.J.; SANCHEZ MERINO, J.M. y cols.: "Penile primary melanoma: Report of a case". Arch. Esp. Urol., 53: 375, 2000.

*3. MILLAN SAGASTE, M.L.; ASENSIO LAHOZ, L.A.; MONGE MIRALLAS, J.M. y cols.: "Melanoma of Penis". Actas Urol. Esp., 27: 152, 2003.

**4. BRACKEN, R.B.; DIOKNO, A.C.: "Melanoma of the penis and uretra". J. Urol., 11: 198, 1974.

5. CASTINEIRAS, J.; VILCHES, J.; LOPEZ, A. y cols.: "Melanoma of glans penis: Report of a case and review of the literature". Arch Esp. Urol., 41: 880, 1998.

6. STILLWELL, T.J.; ZINCKE, H.; GAFFEY, T.A. y cols.: "Malignant melanoma of the penis". J. Urol., 140: 72, 1998.

7. DONATE MORENO, M.J.; RUIZ MONDEJAR, R.; JIMÉNEZ BACHS, J.M. y cols.: "Malignant melanoma of the penis: Report on one case". Arch. Esp. Urol., 58: 672, 2005. 